\title{
First-Order Phase Transition in a Reaction-Diffusion Model With Open Boundary: The Yang-Lee Theory Approach
}

\author{
Farhad H Jafarpour $\dagger$ \\ Bu-Ali Sina University, Physics Department, Hamadan, Iran
}

\begin{abstract}
A coagulation-decoagulation model is introduced on a chain of length $L$ with open boundary. The model consists of one species of particles which diffuse, coagulate and decoagulate preferentially in the leftward direction. They are also injected and extracted from the left boundary with different rates. We will show that on a specific plane in the space of parameters, the steady state weights can be calculated exactly using a matrix product method. The model exhibits a first-order phase transition between a low-density and a high-density phase. The density profile of the particles in each phase is obtained both analytically and using the Monte Carlo Simulation. The two-point density-density correlation function in each phase has also been calculated. By applying the Yang-Lee theory we can predict the same phase diagram for the model. This model is further evidence for the applicability of the Yang-Lee theory in the non-equilibrium statistical mechanics context.
\end{abstract}

PACS numbers: 05.20.-y, 02.50.Ey, 05.70.Fh, 05.70.Ln

Submitted to: J. Phys. A: Math. Gen.

† e-mail:farhad@sadaf.basu.ac.ir 


\section{Introduction}

It is known that one-dimensional driven diffusive systems can exhibit many interesting collective phenomena such as jamming and spontaneous symmetry breaking [1, 2]. Applications of such models are divers and include the kinetics of biopolymerization 3] and traffic flow [4, but to give just a few examples.

A powerful framework for studying equilibrium phase transitions in classical statistical mechanics is the Yang-Lee theory of phase transition 5, 6]. Here we shall briefly explain the key points of this theory. Consider a simple model consisting of $L$ spins in thermal contact with a heat reservoir. The hallmark of a phase transition in this system is the appearance of nonanalyticities in its free energy which can be defined in terms of the partition function of the system $Z_{L}$ as $f=\frac{1}{L} \ln Z_{L}$. In the thermal equilibrium the partition function (which is a function of temperature $T$ in our example) determines the statistical properties of the system. However, since the partition function has no real and positive zeros in the complex- $T$ plane, there is no scope for a phase transition in a finite system. As we increase the number of spins $L$ to infinity, the partition function zeros might accumulate towards a point $T_{0}$ on the real axis so there is the possibility of a phase transition at this point. The density of zeros near the accumulation point determines the order of the phase transition. At a first-order phase transition there is a nonzero density of zeros at the critical point whereas the density of zeros decays to zero at a continuous transition. Although we explained the theory of partition function zeros with reference to a system described by a canonical partition function, we should note that the theory still holds when one is working in grand canonical ensembles. In this case the fugacity (or chemical potential) plays similar role as the temperature $T$. More generally one can look for the zeros of the partition function in the complex plane of any of its intensive variables [7, 8].

The lack of such a theory in the non-equilibrium statistical mechanics context has recently encouraged people to examine whether a generalized version of it can still work for out-of-equilibrium systems. Quite similar to the equilibrium statistical mechanics one can define a generalized partition function as sum over the steady state weights. These weights can be calculated using different methods such as matrix product formalism in which the stationary probability distribution function is written in terms of the products of non-commuting operators [13. Then we look at the zeros of the partition function in the thermodynamic limit in the complex plane of any intensive variable of the model. The zeros might divide the complex plane into several regions where the free energy of the system is analytic. Each region corresponds to a different phase and as we explained above, the order of transition depends on how the zeros accumulate towards the positive real axis. Several out of equilibrium models have been proposed and studied using the generalized version of the Yang-Lee theory [9]-[12, and it seems that the results obtained from application of this theory accord themselves well with the known results obtained from other approaches.

In this paper we will introduce a one-dimensional reaction-diffusion model with open 
boundary and apply the Yang-Lee theory thereto. This model has already been studied with reflecting boundaries [14, 15] while the open boundary problem has not been solved. We shall investigate the effects of the open boundary condition on the phase diagram and also the phase transition points of this model. Our work will also be another example (with more complicated reaction rules) that shows the applicability of the Yang-Lee theory to study the critical behaviors of the out of equilibrium systems.

In chapter (2) we define the model. The chapter (3) is devoted to the application of the matrix product formalism in order to find the steady states weights and therefore the partition function of the model. Using this formalism allows us to find the stationary weights of the model exactly and calculate many interesting quantities such as the density profile of particles on the chain and also the correlation functions. It turns out that our model has a first-order phase transition between a low-density and a highdensity phase provided that there is a constraint on the reaction rates. In chapter (4) we shall apply the Yang-Lee theory to our model and calculate the grand canonical partition function of the system and its line of zeros in the thermodynamic limit. The line of zeros is a circle and the density of zeros is constant over it all. In the Yang-Lee theory language this signifies a first-order phase transition. Finally in section (5) we shall conclude and explain the generalizations.

\section{The Model}

Consider a one-dimensional lattice of length $L$. From the left boundary classical particles are injected with rate $\alpha$ if the target site is empty. They can also be extracted from there with rate $\beta$, provided that it is occupied by a particle. In the bulk of the chain particles diffuse to the left and right. When two of them meet, they can merge into a single particle. Similarly, a single particle can split into two particles. All of these processes take place preferentially to the leftward direction. Specifically, the reaction rules in the bulk of the chain are

$$
\begin{array}{lll}
\text { diffusion to the left: } & \emptyset+A \rightarrow A+\emptyset \text { with rate } q \\
\text { diffusion to the right: } & A+\emptyset \rightarrow \emptyset+A \text { with rate } q^{-1} \\
\text { coagulation at the left: } & A+A \rightarrow A+\emptyset \text { with rate } q \\
\text { coagulation at the right: } & A+A \rightarrow \emptyset+A \text { with rate } q^{-1} \\
\text { decoagulation to the left: } & \emptyset+A \rightarrow A+A \text { with rate } \Delta q \\
\text { decoagulation to the right: } & A+\emptyset \rightarrow A+A \text { with rate } \Delta q^{-1}
\end{array}
$$

in which $A$ and $\emptyset$ represent an occupied and an empty site respectively. At the left boundary we have

$$
\begin{array}{lll}
\text { injection at the first site: } & \emptyset \rightarrow A \text { with rate } \alpha \\
\text { extraction at the first site: } & A \rightarrow \emptyset \text { with rate } \beta .
\end{array}
$$

It is seen that (11) consists of transitions which involve only the configurations of nearest

neighboring sites. For $q>1$ the particles have a tendency to move in the leftward direction. In the following section we will use the Matrix Product Formalism to find the steady states weights of this model. 
First-Order Phase transition in a Reaction-Diffusion Model

\section{Application of the Matrix Product Formalism}

The time evolution operator of our model can be written as

$$
H=\sum_{j=1}^{L-1} h_{j, j+1}+h_{1}^{(L)}
$$

in which

$$
h_{j, j+1}=\mathcal{I}^{\otimes(i-1)} \otimes h \otimes \mathcal{I}^{\otimes(L-i-1)}
$$

and

$$
h_{1}^{(L)}=h^{(L)} \otimes \mathcal{I}^{\otimes(L-1)}
$$

where $\mathcal{I}$ is a $2 \times 2$ identity matrix, $h$ is a $4 \times 4$ matrix for the bulk interactions and $h^{(L)}$ is a $2 \times 2$ matrix for particle input and output at the left boundary. In a basis $(\emptyset \emptyset, \emptyset A, A \emptyset, A A)$ the bulk evolution operator $h$ and $h^{(L)}$ read

$$
h=\left(\begin{array}{cccc}
0 & 0 & 0 & 0 \\
0 & -(\Delta+1) q & q^{-1} & q^{-1} \\
0 & q & -(\Delta+1) q^{-1} & q \\
0 & \Delta q & \Delta q^{-1} & -q+q^{-1}
\end{array}\right), h^{(L)}=\left(\begin{array}{cc}
-\alpha & \beta \\
\alpha & -\beta
\end{array}\right) .
$$

In the following we show that the stationary probability distribution of any configuration of our model can be calculated exactly using the matrix product formalism (MPF) 13 . Let us first briefly review the MPF. According to this approach, for models with Hamiltonian $H=\sum_{j=1}^{L-1} h_{j, j+1}+h_{1}^{(L)}+h_{L}^{(R)}$, the stationary probability distribution $P(\mathcal{C})$ of any configuration $\mathcal{C}$ is assumed to be of the form

$$
P(\mathcal{C})=\frac{1}{Z_{L}}\left\langle W\left|\prod_{i=1}^{L}\left(\tau_{i} D+\left(1-\tau_{i}\right) E\right)\right| V\right\rangle
$$

( $\tau_{i}=0$ if the site $i$ is empty and $\tau_{i}=1$ if it is occupied by a particle) with the following property

$$
H P(\mathcal{C})=0
$$

The factor $Z_{L}$ in (4) is a normalization factor and can easily be obtained using the normalization condition $\sum_{\mathcal{C}} P(\mathcal{C})=1$. The matrices $D$ and $E$ are square matrices and stand for the presence of a particle and an empty site. These matrices beside the vectors $\langle W|$ and $|V\rangle$ satisfy the following algebra

$$
\begin{aligned}
& h\left[\left(\begin{array}{c}
E \\
D
\end{array}\right) \otimes\left(\begin{array}{c}
E \\
D
\end{array}\right)\right]=\left(\begin{array}{c}
\bar{E} \\
\bar{D}
\end{array}\right) \otimes\left(\begin{array}{c}
E \\
D
\end{array}\right)-\left(\begin{array}{c}
E \\
D
\end{array}\right) \otimes\left(\begin{array}{c}
\bar{E} \\
\bar{D}
\end{array}\right), \\
& <W\left|h^{(L)}\left(\begin{array}{c}
E \\
D
\end{array}\right)=-<W\right|\left(\begin{array}{c}
\bar{E} \\
\bar{D}
\end{array}\right), \\
& h^{(R)}\left(\begin{array}{c}
E \\
D
\end{array}\right)\left|V>=\left(\begin{array}{c}
\bar{E} \\
\bar{D}
\end{array}\right)\right| V>.
\end{aligned}
$$


The auxiliary matrices $\bar{E}$ and $\bar{D}$ are also square matrices. For the present model with $h^{(R)}=0$, one can easily see from (3) and (5) that the corresponding algebra is

$$
\begin{aligned}
& {[C, \bar{C}]=[E, \bar{E}]=0} \\
& \bar{E} C-E \bar{C}=\left(q+q \Delta+q^{-1}\right) E C-q(1+\Delta) E^{2}-q^{-1} C^{2} \\
& \bar{C} E-C \bar{E}=\left(q^{-1}+q^{-1} \Delta+q\right) C E-q^{-1}(1+\Delta) E^{2}-q C^{2} .
\end{aligned}
$$

with

$$
\begin{aligned}
& \langle W|((\alpha+\beta) E+\bar{E}-\beta C)=\langle W| \bar{C}=0 \\
& \bar{E}|V\rangle=\bar{C}|V\rangle=0
\end{aligned}
$$

in which we have defined $C:=D+E$ and $\bar{C}:=\bar{D}+\bar{E}$. Traditionally, one should find either a representation for the algebra (6) and (7)/ or calculate the stationary weights (41) directly from the algebra without using any representations. In the following we will show that a finite-dimensional representation of the algebra (6) and (7) exists under special constraint on the parameters $\alpha, \beta, q$ and $\Delta$. One can easily check that for $q^{2} \neq 1+\Delta$ the following matrices and vectors

$$
\begin{aligned}
& C=\left(\begin{array}{cc}
1+\Delta & 0 \\
0 & q^{2}
\end{array}\right), \bar{C}=0, E=\left(\begin{array}{cc}
1 & \lambda \\
0 & q^{2}
\end{array}\right), \bar{E}=\left(\begin{array}{cc}
\frac{q^{2}-1}{q} \Delta & -\frac{\Delta}{q} \lambda \\
0 & 0
\end{array}\right) \\
& |V\rangle=\left(\begin{array}{c}
\frac{\lambda}{q^{2}-1} \\
1
\end{array}\right),\langle W|=\left(\frac{q \Delta\left(q^{2}-q \beta-1\right)}{\lambda(\beta+\beta \Delta-q \Delta)}, 1\right)
\end{aligned}
$$

provide a two-dimensional representation for the algebra (6) and (7) provided that $\alpha=\left(q^{-1}-q+\beta\right) \Delta$. For all rates to be positive we assume $q>1$ and $\beta \geq q-q^{-1}$. For $q^{2}=1+\Delta, C$ is not diagonalizable and we find another representation

$$
\begin{aligned}
& C=\left(\begin{array}{ll}
1 & \lambda \\
0 & 1
\end{array}\right), \bar{C}=0, E=\left(\begin{array}{cc}
\frac{1}{q^{2}} & \lambda \\
0 & 1
\end{array}\right), \bar{E}=\left(\begin{array}{cc}
\frac{\left(q^{2}-1\right)^{2}}{q^{3}} & -\frac{q^{2}-1}{q} \lambda \\
0 & 0
\end{array}\right) \\
& |V\rangle=\left(\begin{array}{c}
\frac{q^{2} \lambda}{q^{2}-1} \\
1
\end{array}\right),\langle W|=\left(-\frac{q^{3}-\beta q^{2}-2 q+q^{-1}+\beta}{\lambda\left(q^{3}-\beta q^{2}-q+\beta\right)}, 1\right) .
\end{aligned}
$$

In (8) and (9), $\lambda$ is a free parameters. Using the representations (8) and (9) one can easily calculate the density profile of the particles in the stationary state and also their density-density correlations. The mean density of particle on the chain at site $i, \rho_{i}$, can be written in terms of the matrices $C$ and $E$ and the vectors $|V\rangle$ and $\langle W|$

$$
\left\langle\rho_{i}\right\rangle=\frac{\left\langle W\left|C^{i-1}(C-E) C^{L-i}\right| V\right\rangle}{\left\langle W\left|C^{L}\right| V\right\rangle} .
$$

By using (8) for $q^{2} \neq 1+\Delta$ and after some algebra we find

$$
\begin{aligned}
& \left\langle\rho_{i}\right\rangle= \\
& \frac{\Delta(1+\Delta)^{-1}\left(1-q^{2}+q \beta\right)\left(\Delta(1+\Delta)^{L} q^{2 i}-\left(q^{2}-1\right)(1+\Delta)^{i} q^{2 L}\right) q^{-2 i+1}}{q \Delta\left(q^{2}-1\right)\left(q^{2 L}-(1+\Delta)^{L}\right)+\beta\left(\Delta q^{2}(1+\Delta)^{L}-q^{2 L}\left(q^{2}-1\right)(1+\Delta)\right)} .
\end{aligned}
$$


Two different phases are apparent: $q^{2}>1+\Delta$ and $q^{2}<1+\Delta$. In the thermodynamic limit $L \rightarrow \infty$ we find two different expressions for the mean density of particles at site $i$. In the first phase, which is called the low-density phase, we obtain

$$
\left\langle\rho_{i}\right\rangle=\frac{q^{2} \Delta\left(q^{-1}-q+\beta\right)}{(1+\Delta)(\beta(1+\Delta)-q \Delta)} e^{-i / \xi} \quad \text { for } \quad q^{2}>1+\Delta .
$$

As can be seen, the density of particles at the left boundary has the largest value and will quickly drop to zero in the bulk of the chain with characteristic length $\xi$ where $\xi^{-1}=\left|\ln \frac{q^{2}}{1+\Delta}\right|$. For the second phase, the high-density phase, we obtain

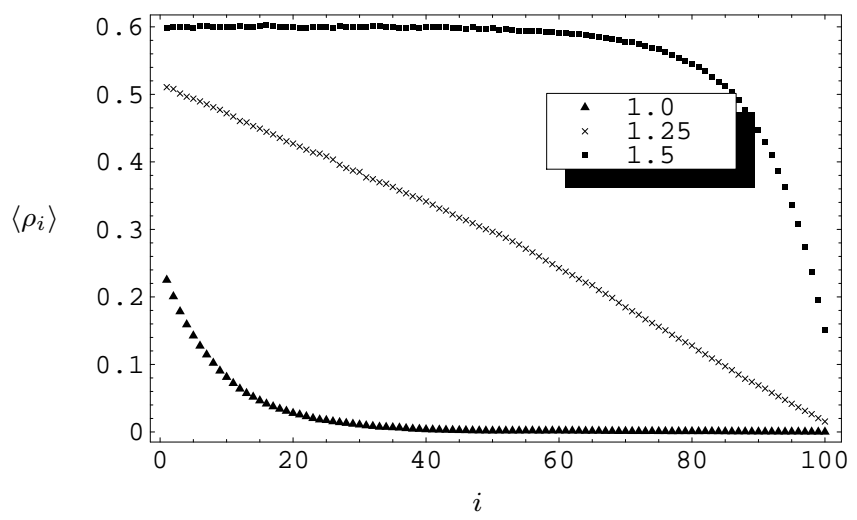

Figure 1. Monte Carlo simulation results for the density profile of the particles on a chain of length $L=100$ for $q=1.5, \beta=0.9$ and different values of $\Delta$. The input rate $\alpha$ is given by $\alpha=\left(q^{-1}-q+\beta\right) \Delta$.

$$
\left\langle\rho_{i}\right\rangle=\frac{\Delta}{1+\Delta}-\frac{q^{2}-1}{1+\Delta} e^{-(L-i) / \xi} \quad \text { for } \quad q^{2}<1+\Delta
$$

where $\xi^{-1}=\left|\ln \frac{q^{2}}{1+\Delta}\right|$. In high-density phase the density of particles is nearly constant in the bulk of the chain equal to $\frac{\Delta}{1+\Delta}$ and drops to zero near the right boundary. For $q^{2}=1+\Delta$ we use (91) to find the following expression for the mean density of particles at site $i$ in the thermodynamic limit

$$
\langle\rho(x)\rangle=\frac{\Delta}{1+\Delta}(1-x) \quad \text { for } \quad q^{2}=1+\Delta
$$

in which $x:=\frac{i}{L}$ and $0 \leq x \leq 1$. In Figure (1) we have plotted the density profile of the particles on a chain of length $L=100$ for different values of $\Delta$ obtained from the Monte carlo simulation. One can readily check that the Monte Carlo results accord with the exact analytical results (12)-(14). In order to see the phase transition more clearly let us define the density of particles averaged over the entire system as an order parameter

$$
\rho:=\frac{1}{L} \sum_{i=1}^{L}\left\langle\rho_{i}\right\rangle
$$

Plot of (15) as a function of $\Delta$ for $q=3, \beta=3$ and two different values of system length $L=200,2000$ is shown in the Figure (2). It can be seen that as the length of the chain approaches the thermodynamic limit $L \rightarrow \infty$, a discontinuity appears in $\rho$ 


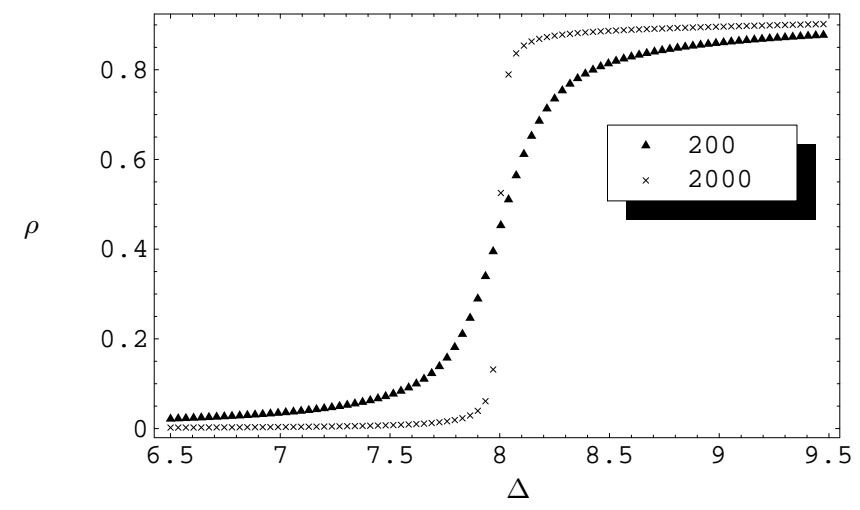

Figure 2. Plot of (15) as a function of $\Delta$ for systems of length $L=200$ and $L=2000$ with $q=3$ and $\beta=3$. In the thermodynamic limit $L \rightarrow \infty$ a first-order phase transition occurs at $\Delta_{c}=q^{2}-1=8$.

at the transition point $\Delta_{c}=q^{2}-1$ which illustrates a first-order phase transition in the system. Using the matrix representation of the algebra (6) and (7) we can also calculate two-point density correlation functions of this model in each phase exactly. The connected two-point density-density correlation function is defined as

$$
\left\langle\rho_{i} \rho_{j}\right\rangle_{c}=\left\langle\rho_{i} \rho_{j}\right\rangle-\left\langle\rho_{i}\right\rangle\left\langle\rho_{j}\right\rangle \text { for } i<j .
$$

As we saw in (10), the density of particles on the chain at an arbitrary site $i$ can be written in terms of the operators $C$ and $E$ and the boundary vectors $\langle W|$ and $|V\rangle$. The expression $\left\langle\rho_{i} \rho_{j}\right\rangle$ can also be written in terms of the same operators and the vectors as

$$
\left\langle\rho_{i} \rho_{j}\right\rangle=\frac{\left\langle W\left|C^{i-1}(C-E) C^{j-i-1}(C-E) C^{L-j}\right| V\right\rangle}{\left\langle W\left|C^{L}\right| V\right\rangle} .
$$

By using the matrix representation of the algebra we find

$$
\left\langle\rho_{i} \rho_{j}\right\rangle=\frac{q \Delta^{2}\left(q^{2}-q \beta-1\right)}{Z_{L}(\beta+\beta \Delta-\Delta q)}\left[\frac{\Delta(1+\Delta)^{L-2}}{q^{2}-1}-q^{2(L-j)}\left(1+\Delta^{j-2}\right)\right]
$$

in which $Z_{L}=\left\langle W\left|C^{L}\right| V\right\rangle$ is given by (201). Now we look for the thermodynamic limit of (16) in each phase. Using (11), (16) and (18) we obtain the following expressions for the thermodynamic limit of the connected two-point correlation function of particles

$$
\left\langle\rho_{i} \rho_{j}\right\rangle_{c}=\left(\frac{\Delta}{1+\Delta}-\left\langle\rho_{i}\right\rangle\right)\left\langle\rho_{j}\right\rangle .
$$

One should use (12) or (13) for $\left\langle\rho_{i}\right\rangle$ in each phase. This expression is valid for both $q^{2}>1+\Delta$ and $q^{2}<1+\Delta$ and has also a simple explanation; namely, in the low-density phase the connected two-point correlation function (16) is non-zero only in the close vicinity of the left boundary where $i<j \sim \xi$. For $j \gg \xi,\left\langle\rho_{i} \rho_{j}\right\rangle_{c} \equiv 0$. Thus, in the whole region $\xi \ll j<L$, particles do not feel any correlations. In the high-density phase both $i$ and $j$ in (16) should be near the last site of the chain far from the left boundary, otherwise $\left\langle\rho_{i} \rho_{j}\right\rangle_{c} \equiv 0$. 


\section{Application of the Yang-Lee Theory}

Now we will examine the Yang-Lee theory. We define the partition function of the system $Z_{L}$ as the sum of the stationary states weights given by (4)

$$
Z_{L}=\sum_{\mathcal{C}} P(\mathcal{C})=\sum_{\left\{\tau_{i}=0,1\right\}}\left\langle W\left|\prod_{i=1}^{L}\left(\tau_{i} D+\left(1-\tau_{i}\right) E\right)\right| V\right\rangle=\left\langle W\left|C^{L}\right| V\right\rangle .
$$

Using the matrix representation of the operator $C$ and the vectors $|V\rangle$ and $\langle W|$ in (8) one obtains

$$
Z_{L}=q^{2 L}+\frac{\Delta q\left(q^{2}-q \beta-1\right)(1+\Delta)^{L}}{\left(q^{2}-1\right)(\beta+\beta \Delta-q \Delta)} .
$$

We look for the zeros of our partition function (20) in the complex- $q$ plane at fixed $\Delta$ and $\beta$ in the thermodynamic limit $L \rightarrow \infty$. In Figure (3) we have plotted the numerical estimates of these roots for $\Delta=8, \beta=3$ and $L=100$. It can be seen that the roots lie on a circle of radius $q_{c}=\sqrt{1+\Delta}=3$, as we had predicted above. The roots also intersect the real- $q$ axis at an angle $\frac{\pi}{2}$ which is the sign of first-order phase transition. Beside the numerical estimates, one can obtain the line of zeros and also their density

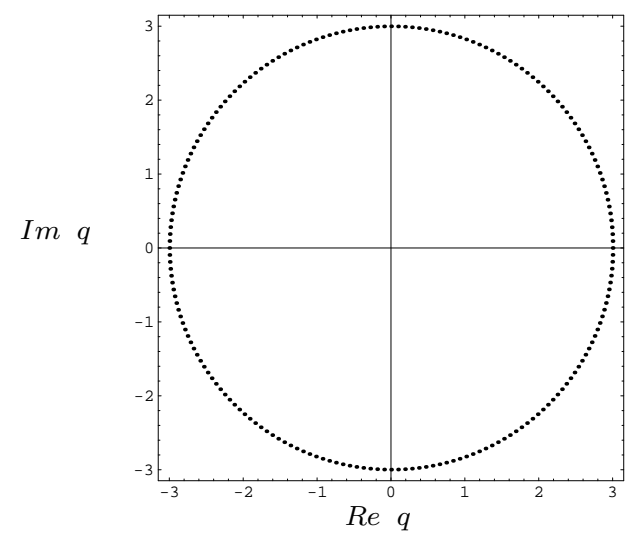

Figure 3. Plot of the numerical estimates for the roots of (20) in the complex $q$ plane for $L=100, \Delta=8$ and $\beta=3$.

near the positive real $q$ axis analytically. Following [7, 8] we define the extensive part of the free energy as

$$
g(q, \Delta, \beta)=\lim _{L \rightarrow \infty} \frac{1}{L} \ln Z_{L}
$$

in which $Z_{L}$ is given by (20). Now the line of zeros can be obtained from

$$
\operatorname{Re} g_{1}=\operatorname{Re} g_{2} \text {. }
$$

Here $g_{1}$ and $g_{2}$ are the thermodynamic limits of $g$ on the left and the right hand side of the phase transition point. By using (20), (21) and (22) and after some calculations one finds the line of zeros

$$
x^{2}+y^{2}=(1+\Delta) .
$$


In (23) the parameters $x$ and $y$ are defined as the real and the imaginary parts of $q$ respectively. This result is in quite agreement with the numerical estimate of zeros given in Figure (3). In order to find the density of zeros $\mu$ near the positive real $q$ axis, we use [7, 8]

$$
2 \pi \mu(s, \Delta, \beta)=\frac{\partial}{\partial s} \operatorname{Im}\left(g_{1}-g_{2}\right)
$$

where $s$ is the arc length of the line of zeros which is measured from the critical point and increases along with the line of zeros. The functions $g_{1}$ and $g_{2}$ have the same definitions as mentioned above. After some algebra we find $\mu=\frac{1}{\pi \sqrt{1+\Delta}}$. It is seen that the density of zeros is constant everywhere on the circle beside in the vicinity of the positive real $q$ axis; therefore, it confirms the existence of a first-order phase transition in our model.

\section{Concluding Remarks}

In this paper we introduced a one-dimensional out-of-equilibrium model consisting of one class of particles which diffuse, coagulate and decoagulate on an open chain. The left boundary of the chain is assumed to be open so that the particles can enter or leave the system. Exact calculations using the MPF show that on a specific plane in the parameters space a first-order phase transition takes place between a low-density and a high-density phase. The density profile of the particles in each phase is obtained exactly. In the low-density phase the density profile of particles far from the left boundary drops exponentially to zero with the length scale $\xi=\left|\ln \frac{q^{2}}{1+\Delta}\right|^{-1}$. We should mention that in the stationary state there exists only one characteristic length scale $\xi$, while the same model without injection and extraction of particles has three different length scales 14, 15]. In the high-density phase the density of particles is nearly constant in the bulk; however, it drops to zero near the right boundary with the same length scale $\xi$. It is shown that the steady state of our model can be written in terms of the superposition of shocks [16]. In order to answer the question whether we can apply the classical Yang-Lee theory to the out-of-equilibrium systems such as the one introduced here, we have seen that the application of this theory gives same results obtained from the MPF approach. We calculated the line of partition function zeros exactly. It intersects the positive real

$q$ axis at right angles at $q_{c}=\sqrt{1+\Delta}$. The density of zeros is uniform at the cross point, which means a first-order phase transition at critical point. Our model, which is more complex than those studied before, is another example for the generality of the classical Yang-Lee picture of phase transitions in one-dimensional out-of-equilibrium models. It can be shown that the MPF also works when the particles are allowed to leave the chain from the right boundary; however, there should be still a constraint on the reaction rates. The whole phase diagram of this model which can be studied at the mean field level or using the Monte-Carlo simulation, is still an open problem. 


\section{References}

[1] Schmittmann B and Zia R K P 1995 Phase transitions and critical phenomena, vol. 17, eds. C. Domb and J. Lebowitz (Academic Press, London).

[2] Schütz G M 1999 Phase transitions and critical phenomena, vol. 19, eds. C. Domb and J. Lebowitz (Academic Press, New York).

[3] McDonald C T, Gibbs J H and Pipkin A C 1968 Biopolymers 6, 1.

[4] Chowdhury D, Santen L and Schadschneider A 2000, Phys. Rep. 329199.

[5] Yang C N and Lee T D 1952 Phys. Rev. 87404.

[6] Yang C N and Lee T D 1952, Phys. Rev. 87410.

[7] Grossmann S and Rosenhauer W 1969 Z. Phys. 218437.

[8] Grossmann S and Lehmann V 1969 Z. Phys. 218449.

[9] Jafarpour F H cond-mat/0209558.

[10] Arndt P F 2000 Phys. Rev. Lett. 84814.

[11] Blythe R A and Evans M R 2002 Phys. Rev. Lett. 89080601.

[12] Dammer S M, Dahmen S R and Hinrichsen H 2002 J. Phys. A: Math. Gen. A 354527.

[13] Derrida B, Evans M R, Hakim V and Pasquier V 1993 J. Phys. A: Math. Gen. A 261493.

[14] Hinrichsen H, Krebs K and Peschel I 1996 Z. Phys. B 100105.

[15] Hinrichsen H, Krebs K and Peschel I 1996 J. Phys. A: Math. Gen. A 292643.

[16] Krebs K, Jafarpour F H and Schütz G M, In preparation. 